\title{
Efectos de un programa de intervención para aumentar la reflexividad y la planificación en un ámbito escolar de alto riesgo por pobreza*
}

Effects of a training program to increase reflexivity and planning in a school area at risk due to poverty

Recibido: septiembre 17 de 2009 ～Revisado: diciembre 13 de 2009 Aceptado: julio 1 de 2010

\author{
VANESSA ARÁN-FILIPPETTI ** \\ María CRISTINA RichaUd de Minzi*** \\ Centro Interdisciplinario de Investigaciones en Psicología \\ Matemática y Experimental, Buenos Aires, Argentina
}

\section{RES UMEN}

El propósito de este estudio fue analizar las diferencias en el estilo cognitivo reflexividad-impulsividad (R-I) y en la capacidad de planificación según el riesgo social, y poner a prueba la eficacia de un programa de intervención integrado a la tarea curricular áulica en un contexto de pobreza. Se trabajó con una muestra de 110 niños de 6 años de edad: experimental $(N=47)$ y control $(N=22)$ en riesgo por pobreza y un grupo control sin riesgo $(N=$ 41) pertenecientes a Entre Ríos, Argentina. Los resultados indican claras diferencias en el patrón de respuestas R-I y en la planificación según el riesgo social, lo que apunta al papel de la experiencia en el desarrollo de estas funciones. Además brindan apoyo a la hipótesis que supone la posibilidad de aumentar la disposición reflexiva mediante un entrenamiento sistematizado. Palabras clave autores

Intervención, reflexividad, planificación, pobreza.

Palabras clave descriptores

Psicología social, Psicología experimental, Psicosociología de la educación, Relación escuela-comunidad.

\section{A B S T R A C T}

The aim of this paper was to analyze the differences Reflexivity-Impulsivity in cognitive style and planning as regards social risk and to test the efficacy of a reflexivity training program integrated to the curriculum in a poverty context. We have worked with a sample of 1106 -year-old children: an experimental group $(N=47)$ a control group $(N=22)$ at risk and a control group not at risk $(N=41)$. All of them live in Entre Ríos, Argentina. The results indicate differences in R-I response patterns and planning in terms of social risk and emphasize the role of experience in the development of these functions. Besides, they support the hypothesis which holds the possibility to improve the reflexive disposition after program training.

Key words authors

Intervention, Reflexivity, Planning, Poverty.

Key words plus

Social Psychology, Experimental Psychology, Educational Psychosociology, School Community Relationship. 


\section{Introducción}

En los últimos años, los estudios en torno al estilo cognitivo reflexividad-impulsividad (en adelante R-I) han experimentado un notable auge, debido a su demostrada relación con el aprendizaje escolar. El concepto fue acuñado en la década de los 60 por Kagan et al. (Kagan, Rosman, Day, Albert \& Phillips, 1964), para hacer referencia al modo particular con el que un niño se enfrenta a tareas que implican incertidumbre de respuesta, y se analiza en función de un constructo bipolar que va desde la reflexividad hasta la impulsividad. Se supone que los individuos reflexivos serían más capaces, al momento de enfrentarse a tareas que plantean incertidumbre, ya que emplean estrategias de análisis y recuerdo que les permite valorar diferentes alternativas de respuesta, convirtiéndolos en sujetos más eficaces para monitorear sus respuestas y adaptarse a las exigencias del medio. Por el contrario, los sujetos impulsivos presentarían dificultades para resolver tareas por la precipitación de respuestas, un menor control atencional y un uso inadecuado de estrategias de tipo analítico. En este sentido, las ventajas del estilo reflexivo en el ámbito académico son evidentes, debido a que las tareas educativas requieren principalmente de un procesamiento analítico y secuencial. Además, se ha sugerido que el estilo cognitivo actuaría como variable moderadora entre los recursos del individuo y sus logros académicos y cognitivos.

Definida en función del constructo R-I, la impulsividad cognitiva o del procesamiento de la información, se relaciona con aspectos cognitivos como el afrontamiento y resolución de problemas (Servera Barceló \& Galván Pascual, 2001). Se ha sugerido que la R-I depende de diversos factores biológicos, psicológicos y culturales. Si bien las evidencias empíricas a favor de la hipótesis que postula el papel del ambiente, son diversas, aún no queda del todo claro el papel que cumpliría en el desarrollo de estrategias analíticas y su asociación con otros factores cognitivos y psicológicos. Un estudio reciente demostró una asociación entre el maltrato infantil y la impulsividad cognitiva (Fernández-Millán, Pérez-Mañez \& Carrasco
Salmerón, 2002) lo que demuestra, de alguna manera, cómo la experiencia recibida puede influir en la R-I de las personas. A favor de esta hipótesis, Olson, Bates y Bayles (1990) han indicado como la interacción cognitiva parental-hijo se asocia con capacidades futuras de control de impulsividad y autorregulación.

El proceso de planificación implica la posibilidad de anticipar consecuencias, generar y seleccionar alternativas, tomar decisiones y sostener la atención (Lezak, 1995). Asimismo, es necesario un adecuado control de la impulsividad y memoria de trabajo. En este sentido, la planificación se relaciona con el estilo reflexivo ya que supone procesos cognitivos compartidos como un mayor control atencional, memoria de trabajo y uso de un lenguaje autodirigido que permita autorregular la conducta.

Desde el punto de vista funcional, la planificación depende de la corteza prefrontal dorsolateral derecha (Unterrainer et al., 2004) y sigue un desarrollo paralelo a la maduración de los lóbulos frontales. Se ha indicado que el estilo parental, el vínculo paterno y las estrategias disciplinarias empleadas cumplen un papel importante en el desarrollo de la autorregulación en los niños (Winsler, Díaz, McCarthy, Atencio \& Chabay, 1999): capacidad ligada al desarrollo de la planificación. A su vez, estudios empíricos aportan evidencia sobre la importancia de la interacción verbal adulto/niño en el desarrollo del sentido de temporalidad (Hudson, 2002) y en la capacidad de planificación (Gauvain, 2001; Radziszewska \& Rogoff, 1988). Esto indica como el desarrollo de la planificación se produce en interacción con los otros y se construye socialmente. Numerosos autores apuntan al carácter cultural y social de la planificación (Baker-Sennet, Matussov \& Rogoff, 1992, 1993; Gauvain, 2001), de manera que un ambiente desfavorecedor con escasas interacciones verbales podría condicionar el desarrollo de esta función.

Respecto al contexto y el desempeño cognitivo, se ha indicado que vivir en la pobreza influye en el desarrollo cognitivo y socioemocional y en el desempeño obtenido en tests cognitivos (Aber, Bennet, Conley \& Li, 1997). Asimismo, se ha 
señalado cómo los niños en condiciones de pobreza suelen presentar menores habilidades relacionadas con la escuela en relación con niños de clase media, progresos escolares más lentos y una mayor deserción escolar (McLanahan, Astone \& Marks, 1991; Ramey \& Campbell, 1991). Más recientemente, desde el paradigma de las neurociencias cognitivas, se han indicado algunas diferencias bioquímicas y neurofuncionales asociadas al nivel socioeconómico.

Existen diversos factores ambientales, más allá de la desnutrición, que pueden afectar los procesos de desarrollo cerebral. Uno de los más significativos es el producido por el estrés, ya que activa la corteza suprarrenal que secreta hormonas esteroideas como el cortisol (Larson, Gunnar, \& Hertsgaard, 1991; Lewis \& Thomas, 1990). Los niveles elevados y permanentes de cortisol afectan al funcionamiento cerebral y cognitivo (Flinn \& England, 1997). Dado que estudios previos han demostrado que los niños de estrato socioeconómico bajo presentan niveles más elevados de cortisol, respecto a niños de estrato medio (Lupien, King, Meaney, \& McEwen, 2001) y obtienen un desempeño cognitivo inferior en tareas ejecutivas sensibles al funcionamiento prefrontal (Farah et al., 2006; Lipina, Martelli, Vuelta, Injoque-Ricle, \& Colombo, 2004; Noble, Norman, \& Farah, 2005), el estrés podría actuar como un importante mediador de la asociación entre el estrato socioeconómico y el desempeño cognitivo.

Por otra parte, los circuitos que están involucrados en la regulación de la emoción son altamente interactivos con los que están asociados a las funciones ejecutivas (tales como planificación, juicio y toma de decisiones), que están íntimamente involucradas en el desarrollo de las habilidades, para la resolución de problemas durante los años del preescolar (Posner \& Rothbart, 2000). En términos del funcionamiento básico del cerebro, las emociones sostienen a las funciones ejecutivas cuando están bien reguladas, pero interfieren con la atención y la toma de decisiones cuando están controladas pobremente (Bush, Luu, \& Posner, 2000; Damasio, 1999; Davis, 1992; LeDoux, 1996; Shonkoff \& Phillips, 2000).
En función de lo expuesto, es posible inferir que el contexto ambiental y la experiencia recibida, actuando a través de factores sociales, cognitivos y emocionales mediadores, podrían inducir diferencias respecto al modo de procesar la información y en la elaboración de planes eficaces. En este sentido, el propósito de la presente investigación es buscar evidencia que aporte a la comprensión de la influencia social en el patrón de respuesta R-I y en la capacidad de planificación, y obtener datos respecto a la posibilidad de aumentar la disposición reflexiva y la planificación mediante estrategias adaptadas al currículo escolar en un ámbito de alto riesgo por pobreza.

Diversos autores han planteado la posibilidad de aumentar la reflexividad en alumnos con impulsividad (Miranda, Presentación \& Jarque, 1999; Miranda, Roselló \& Soriano, 1998) y han señalado la eficacia de los programas de intervención integrados a la tarea áulica (Gargallo, 1993a, 1993b), aunque los mismos se han centrado fundamentalmente en poblaciones escolares sin riesgo o grupos con trastornos clínicos específicos. En el momento, persiste la escasez de estudios respecto al éxito de los programas de intervención para aumentar la reflexividad en poblaciones en riesgo por pobreza.

Los procedimientos utilizados que han resultado eficaces para reducir la impulsividad, combinan técnicas cognitivas y cognitivo-conductuales. Entre los más utilizados se encuentra el método de autoinstrucciones de Meichenbaum y Goodman (1971), que mediante una serie de pasos específicos busca promover la autorregulación de la conducta y favorecer el pensamiento reflexivo. Otras técnicas efectivas incluyen el modelado participativo, estrategias de escudriñamiento o análisis cuidadoso, demora forzada y uso de reforzadores, entre otras (Gargallo, 1993a).

Con base en lo expuesto previamente, el presente estudio intentará dar respuesta a los siguientes interrogantes: iLos contextos de pobreza pueden provocar patrones de respuesta diferenciales en el constructo R-I? Por otra parte, iLos niños en situación de vulnerabilidad social presentan menores habilidades de planificación que los no vulnerables? Y, por último, iEs posible aumentar 
la disposición reflexiva y la capacidad de planificación mediante el empleo de estrategias cognitivas adaptadas al currículo escolar?

\section{Método}

\section{Sujetos}

Dentro de un programa mayor ${ }^{1}$, el presente estudio sigue un diseño de investigación antes-después, con doble grupo control (con y sin riesgo). Se trabajó con una muestra de 110 niños de ambos sexos, de 6 años de edad, que asisten a dos escuelas de riesgo por pobreza y una de clase media de la ciudad de Paraná (Entre Ríos-Argentina). La primera escuela definida en riesgo es una escuela parroquial sostenida por el Arzobispado de Paraná y el estado provincial y fue seleccionada por el Consejo General de Educación de la provincia de Entre Ríos. La segunda escuela en riesgo se seleccionó entre los establecimientos educativos incluidos en el Plan Nacional de Mil Escuelas Bajo el Nivel de Pobreza, de acuerdo al programa Integral para la Igualdad Educativa (PIIE), dependiente del Ministerio de Educación del la República Argentina. Los niños que concurren a estas dos escuelas viven en barrios marginales de la ciudad de Paraná, con sus necesidades básicas insatisfechas o apenas satisfechas, viviendas precarias, con padres con escolaridad primaria o que no han alcanzado a finalizarla, que están desempleados, subempleados o con empleos mal calificados. La mayoría de estos niños realiza trabajos como cartonero, abrir puertas de autos, llevar las compras del supermercado a señoras, etc., con lo cual pasan mucho el tiempo en la calle a la vez que faltan mucho a la escuela, si no es que desertan. Muchas de estas familias presentan problemas socioafectivos de distinta gravedad, debido en gran parte a las dificultades que deben sobrellevar.

La tercera escuela no en riesgo, fue seleccionada al azar entre las escuelas primarias de Paraná,

1 "Sin afecto no se aprende ni se crece. Un programa para reforzar los recursos cognitivos, afectivos y lingüísticos de niños en riesgo ambiental por pobreza extrema”, dirigido por la Dra. María Cristina Richaud. instaladas en barrios de clase media, con niños cuyos padres tienen en promedio nivel educacional secundario, son empleados, pequeños comerciantes o profesionales universitarios.

\section{Grupo 1}

Niños en condiciones de riesgo ambiental con intervención. 47 niños de ambos sexos de 6 años de edad que asisten a una escuela urbano-marginal y pertenecen a la categoría de alto riesgo.

\section{Grupo 2}

Niños en condiciones de riesgo ambiental sin intervención (Control con riesgo), 22 niños que asisten a una escuela urbano-marginal y que pertenecen a la categoría de alto riesgo. Muestra accidental apareada por edad, género y nivel de instrucción. Esta escuela se seleccionó teniendo en cuenta que la caracterización de la población y las características del establecimiento educativo fueran similares a las del grupo 1 .

\section{Grupo 3}

Niños no en riesgo (Control sin riesgo), 41 niños. Muestra accidental apareada por edad, género y nivel de instrucción. Sujetos sin antecedentes clínicos neurológicos ni psiquiátricos, que cursan sus estudios escolares con regularidad. Sin repitencia escolar y sin necesidad de estudios pedagógicos correctivos. Concurren a una escuela de barrio de clase media.

\section{Procedimiento ético}

Para trabajar con los niños se solicitó una entrevista con los directivos de las escuelas a quienes se explicaron las características de la investigación. Posteriormente, se envió a los padres y a las madres una nota explicándoles los objetivos del trabajo y la tarea que se desarrollaría con los niños y niñas. Se les aclaró expresamente que la colaboración era absolutamente voluntaria y anónima, con el compromiso de no proporcionar ninguna información 
individual al personal de la escuela, a excepción de aquellos casos en que fuera requerida especialmente por los padres y madres. Finalmente, se obtuvo el permiso escrito de todos los padres y madres antes de comenzar con la experiencia.

\section{Instrumentos}

Se administraron los siguientes instrumentos, en el orden presentado, dentro del ámbito escolar.

Test de emparejamiento de figuras conocidas (MFF-20, por sus siglas en inglés)

El MFF-20 es una prueba de emparejamiento perceptiva aplicable en niños de 6 a 12 años de edad. Evalúa el constructo reflexividad-impulsividad que está relacionado con la capacidad del niño para solucionar tareas definidas por la incertidumbre. Consta de dos ítems iniciales de ensayo y 20 ítems-evaluación. La prueba consiste en presentar al niño simultáneamente un dibujo modelo y seis opciones diferentes de respuestas de las cuales una sola es la correcta. El niño debe seleccionar la figura idéntica al modelo, para lo cual dispone de seis oportunidades. Las variables que se investigan son: la cantidad de errores y el tiempo de latencia. Con base en un cálculo que se obtiene combinando ambas variables, es posible obtener indicadores para la impulsividad y la ineficiencia.

El MFF-20 fue diseñado por Cairns y Cammock (1978) con el objetivo de aumentar los índices psicométricos de la versión anterior. Los estudios sobre el MFF-20 suponen mejores índices de confiabilidad y validez en relación al MFFT original, lo que hacen de este test el instrumento más importante de evaluación del la R-I (Buela-Casal, Carretero-Dios \& De los Santos-Roig, 2001). La fiabilidad del test utilizando el método de la división por las dos mitades con la corrección de Spearman-Brown es de 0.89 para los errores y de 0.91 para las latencias; los coeficientes de estabilidad para latencias y errores fueron de 0.85 y 0.77 , respectivamente, y la correlación negativa entre errores y latencias dependiendo de la edad, osciló entre -0.62 y -0.67 (Cairns \& Cammock, 1978).

\section{Test de laberintos (WPPSI)}

Este es un subtest de la escala de inteligencia de Wechsler de preescolar y primaria (WPPSI por sus siglas en inglés) que ofrece una medida de la coordinación visomotriz, de la organización perceptual, del razonamiento no verbal y de la capacidad de planificación. La tarea consiste en trazar una línea desde el inicio de cada laberinto hasta la salida, sin levantar el lápiz, sin entrar en pasajes sin salida o traspasar las paredes del mismo. Consta de 10 laberintos de complejidad creciente y cada laberinto tiene un tiempo límite para su correcta ejecución.

\section{Procedimiento de intervención}

Se aplicó un programa para aumentar la reflexividad y la capacidad de planificación en función de los siguientes criterios:

\section{Entorno social}

Para promover la reflexividad y la autorregulación se incrementó el la regulación verbal externa y la supervisión de la conducta por medio de la propuesta de reglas simples y claras, la técnica del modelado para favorecer el uso de un lenguaje autodirigido, la enseñanza de estrategias reflexivas y el uso de reforzadores positivos mediante un sistema continuo de puntos.

El interventor y luego el profesor impartieron las consignas y ejemplificaron la realización de los ejercicios en el pizarrón, verbalizando los pasos necesarios para su correcta ejecución. Posteriormente, las tareas fueron realizadas por los niños sin regulación verbal externa. Al finalizar los ejercicios, se verificó en forma conjunta la ejecución de los mismos y se ofreció la posibilidad de autocorrección. Por último, cada niño con ayuda del interventor y/o profesor asignó la puntuación al ejercicio y, de acuerdo a la misma, avanzó un casillero de un camino final, a modo de recompensa con aprobación social. 
Lugar y tiempo de intervención

La intervención se llevó a cabo en el mismo centro educativo, a razón de 3 a 4 sesiones semanales de 30 minutos cada una. El programa se aplicó durante aproximadamente tres meses, tiempo tras el cual se evalúo nuevamente la planificación y la Reflexividad-Impulsividad cognitiva, para comparar las diferencias de funcionamiento cognitivo pre y posintervención. El tiempo se determinó en función de lograr la implementación de 35 sesiones en el ámbito escolar. La estructuración de programas con sesiones de similar cantidad ( 27 a 30 sesiones) han sido empleados en estudios previos, para analizar los cambios pre y posintervención respecto a la posibilidad de aumentar la reflexividad en el aula (Gargallo, 1993a) con resultados positivos. Por otro lado, consideramos que la evaluación de los resultados de los programas no se debería extender más allá del tiempo sugerido, para asegurarse que los cambios se produzcan como producto de la intervención y no como consecuencia del efecto del tiempo y la maduración sobre estas funciones.

La evaluación posintervención se aplicó únicamente en el grupo experimental en riesgo, debido a que se consideró que no existen fundamentos como para esperar que en el lapso establecido las funciones cognitivas estudiadas se modificaran por efecto de la maduración cerebral. Por otro lado, los niños pertenecientes al grupo control sin riesgo en la evaluación pretest, ya tenían un estilo cognitivo reflexivo y la cantidad de errores y el tiempo de latencia eran los esperados de acuerdo a la edad cronológica, por lo que no era esperable un aumento de reflexividad. Asimismo, se señaló como limitación la posibilidad de obtener un permiso para que la escuela permitiera evaluar nuevamente a los niños en ambas escuelas controles.

\section{Naturaleza de la tarea}

Los ejercicios empleados fueron sencillos y las consignas simples y claras. Los mismos fueron diseñados con el objetivo de plantear incertidumbre en las respuestas y estimular los tiempos de latencia, de modo de favorecer el uso de estrategias analíticas y el estilo reflexivo.

\section{Estrategias de intervención}

Se diseñaron cuadernos de intervención denominados P.E.C.E., Programa de Estimulación Cognitivo Escolar (Arán, Filippetti \& Richaud de Minzi, 2008), para estimular las funciones cognitivas básicas adaptadas al medio escolar. Debido a que el objetivo final fue realizar una intervención con criterios ecológicos que se adaptaran a las necesidades áulicas y a la tarea educativa, los ejercicios del cuaderno se ofrecieron como modelo y los interventores, trabajando en forma conjunta con el profesor, diseñaron sus propias estrategias en función de los ejercicios base, pero adaptadas al currículo escolar.

La intervención para aumentar los recursos cognitivos y la reflexividad se fundamentó en el uso de las siguientes estrategias:

1. Modelado: al inicio de cada actividad se verbalizaron los pasos necesarios para resolver las actividades en el aula. Este procedimiento fue realizado primero por el interventor y/o profesor y luego por los niños.

2. Autoinstrucciones: este método se fundamenta en el papel del lenguaje como regulador de la conducta (Luria, 1961). Se entrenó a los niños para que utilizaran una serie de pasos durante la ejecución de los ejercicios, similares a los del procedimiento diseñado por Meichenbaum y Goodman (1971). Mediante esta técnica, se procura enseñar a los niños a utilizar el lenguaje interno para regular la conducta y favorecer de este modo la autorregulación.

3. Enseñanza de estrategias de escaneo y discriminación visual cuidadoso: estas técnicas han demostrado ser unas de las más eficaces en el entrenamiento de la reflexividad (Egeland, 1974; Meichenbaum \& Goodman, 1971). Para estimular el empleo de estas estrategias, se utilizaron los siguientes procedimientos: 
- Ejercicios de búsqueda de figuras semejantes a un modelo: mediante tareas de emparejamiento similares a las del paradigma del MFF (Kagan, 1965), se le enseñó al niño a emplear estrategias analíticas para seleccionar dentro de una serie de variantes similares, la figura idéntica al modelo.

- Ejercicios de atención selectiva y de búsqueda de semejanzas y diferencias entre figuras: mediante estos ejercicios se le enseñó al niño a mirar y comparar figuras similares y a realizar un análisis cuidadoso de los estímulos presentados para seleccionar las respuestas correctas y eliminar las incorrectas.

4. Ejercicios de planificación: para estimular la planificación se utilizaron estrategias que implican anticipar y monitorear pasos y el uso de diferentes recursos para lograr un objetivo (secuenciación, laberintos de complejidad creciente, ordenamiento de figuras). Asimismo, debido al carácter social de la planificación (Baker-Sennet, Matussov \& Rogoff, 1992, 1993; Gauvain, 2001), los ejercicios se realizaron primero conjuntamente entre el adulto y los niños y, posteriormente, lo hizo el propio niño.

5. Uso de reforzadores: se empleó un sistema continuo de puntos, a modo de recompensa con aprobación social.

\section{Procedimientos estadísticos}

Para describir perfiles neurocognitivos según vulnerabilidad social, se realizó análisis de varianza multivariado (MANOVA), incorporando la variable escuela según riesgo como factor fijo y las variables impulsividad, errores, latencia y planificación como variables dependientes. Además se empleó MANOVA para medidas repetidas, con el objetivo de conocer las diferencias pre y posintervención en el grupo experimental. El procesamiento y análisis estadístico de los datos se realizó utilizando el Statistical Package for the Social Sciences (SPSS) versión 11.5.

\section{Resultados}

Comparación de las variables cognitivas en grupos con y sin vulnerabilidad social antes de la intervención

En la Tabla 1 se presentan las medias aritméticas y contrastes post hoc para los indicadores impulsividad, errores, tiempo de latencia y planificación, que obtienen el grupo experimental y control en situación de vulnerabilidad social, y el grupo control sin riesgo.

El MANOVA indicó una diferencia estadísticamente significativa en general entre los grupos $(F(8,206)$ Hotelling $=10.161 ; p<0.000)$. Específicamente, estas diferencias se encontraron en

\section{TABLA 1}

Comparación de puntajes obtenidos respecto a la impulsividad y la planificación entre niños con y sin vulnerabilidad social antes de la intervención

\begin{tabular}{|c|c|c|c|c|c|c|c|c|c|c|c|}
\hline \multirow{2}{*}{ Funciones } & \multicolumn{2}{|c|}{$\begin{array}{c}\text { Riesgo } \\
\text { experimental }\end{array}$} & \multicolumn{2}{|c|}{$\begin{array}{l}\text { Control con } \\
\text { riesgo }\end{array}$} & \multicolumn{2}{|c|}{$\begin{array}{l}\text { Control sin } \\
\text { riesgo }\end{array}$} & \multirow{2}{*}{$\begin{array}{c}F \\
(2,107)\end{array}$} & \multirow{2}{*}{$P$} & \multirow{2}{*}{ M1-M2 } & \multirow{2}{*}{ M1-M3 } & \multirow{2}{*}{ M2-M3 } \\
\hline & M1 & DE1 & M2 & DE2 & M3 & DE3 & & & & & \\
\hline Impulsividad & 1.75 & 1.387 & 1.71 & 1.46 & -0.0056 & 1.53 & 18.476 & 0.000 & 1.000 & 0.000 & 0.000 \\
\hline - Errores & 43.40 & 9.006 & 39.91 & 10.65 & 31.71 & 9.188 & 17.266 & 0.000 & 0.461 & 0.000 & 0.004 \\
\hline - Latencia & 112.44 & 69.37 & 100.153 & 72.37 & 192.60 & 121.33 & 10.703 & 0.000 & 1.000 & 0.000 & 0.001 \\
\hline Planificación & 14.09 & 6.290 & 16.18 & 4.992 & 22.34 & 2.565 & 31.749 & 0.000 & 0.309 & 0.000 & 0.000 \\
\hline
\end{tabular}

Fuente: elaboración propia 
cuanto a la impulsividad cognitiva $(F(2,107)=$ 18.476 ; $p<0.000)$, la cantidad de errores $(F(2$, $107)=17.266 ; p<0.000)$, al tiempo de latencia $(F(2,107)=10.703 ; p<0.000)$ y la planificación $(F(2,107)=31.749 ; p<0.000)$ (véanse Tabla 1 y Figura 1$)$.

Efectos de la intervención sobre las funciones cognitivas en el grupo experimental

El MANOVA de medidas repetidas para el análisis de la influencia de la intervención sobre el estilo cognitivo R-I (MFF-20) y la capacidad de planificación (WISC-R), demostró diferencias significativas en el antes-después de la intervención ( $F(4$, 43) Hotelling $=31.138 ; p<0.000$ ). Los análisis univariados indican diferencias estadísticamente significativas en cuanto a la impulsividad cognitiva $(p<0.000)$, la cantidad de errores $(p<0.000)$ y el tiempo de latencia $(p<0.000)$ del MFF-20 y la capacidad de planificación $(p<0.000)$ (véase Tabla 2).

\section{Comparación de las variables cognitivas en grupos con y sin vulnerabilidad social después de la intervención en el grupo experimental}

Cuando se compara el desempeño entre los grupos después de la intervención, se evidencian diferencias significativas entre los grupos $(F(8,206)$ Hotelling $=9.367 ; p<0.000)$. Los contrastes post hoc

FIGURA 1

Puntajes medios obtenidos respecto a los errores y latencia de la prueba de R-I (MFF-20) y el test de laberintos (WPPSI) antes de la intervención según grupos

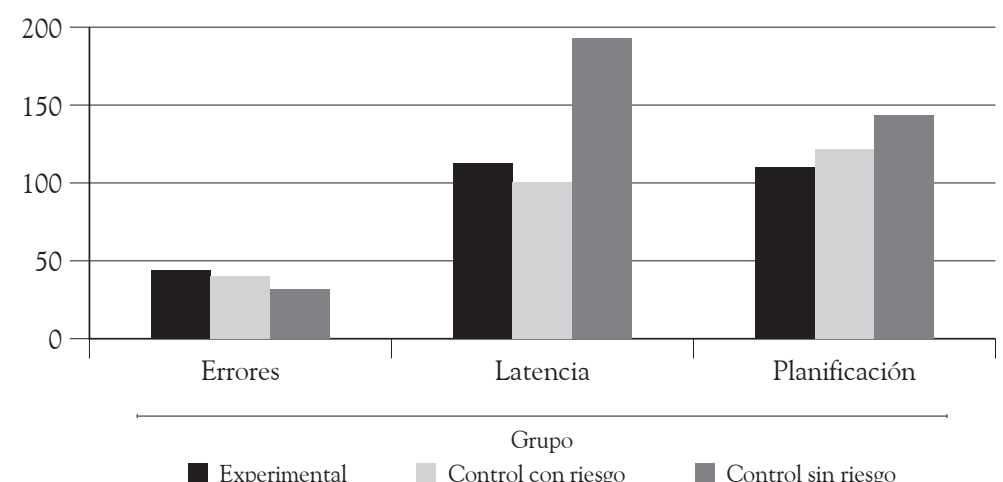

Fuente: elaboración propia

TABLA 2

Medias aritméticas, desviaciones estándar y F correspondientes a los valores obtenidos en Impulsividad, Errores, Latencia y Planificación antes y después de la intervención en el grupo en riesgo experimental

\begin{tabular}{|c|c|c|c|c|c|c|}
\hline \multirow{3}{*}{ Funciones } & \multicolumn{6}{|c|}{ Fase de intervención } \\
\hline & \multicolumn{2}{|c|}{ Pre } & \multicolumn{2}{|c|}{ Post } & \multirow{2}{*}{$F(1,46)$} & \multirow{2}{*}{$p$} \\
\hline & $\mathbf{M}$ & $\mathrm{DE}$ & $\mathrm{M}$ & $\mathrm{DE}$ & & \\
\hline Impulsividad cognitiva & 1.75 & 1.38 & -0.8968 & 1.89 & 90.295 & 0.000 \\
\hline - Errores & 43.40 & 9.01 & 28.96 & 12.821 & 56.962 & 0.000 \\
\hline - Latencia & 112.44 & 69.37 & 286.86 & 120.92 & 90.521 & 0.000 \\
\hline Planificación & 14.09 & 6.29 & 20.87 & 4.199 & 73.455 & 0.000 \\
\hline
\end{tabular}

Fuente: elaboración propia 
revelan que el grupo que recibió la intervención, comete menos errores $(p<0.001)$, emplea tiempos de latencia mayores $(p<0.000)$ y obtiene un desempeño superior respecto a la planificación $(p$ $<0.000$ ) en relación con los niños que no recibieron intervención. Además, el grupo experimental no se diferencia después de la intervención del grupo control sin riesgo en las variables errores y planificación, por lo que se asume que luego de la intervención, el grupo experimental aumentó la reflexividad y la capacidad de planificación alcanzando un nivel similar al grupo control sin riesgo. Asimismo, se evidencia una diferencia significativa respecto al tiempo de latencia, pero a favor del grupo experimental, es decir que los niños que recibieron la intervención emplean un tiempo aún mayor en relación al grupo control sin riesgo (véase Tabla 3).

\section{Discusión}

Los datos del presente estudio aportan evidencia empírica a la hipótesis que apunta al papel del contexto ambiental en el desarrollo de estrategias analíticas y en la capacidad de planificación. Los resultados indican que el estilo R-I se encuentra asociado a la vulnerabilidad social, lo que sugiere que el estilo cognitivo de los niños estaría explicado, en parte, por la estimulación verbal, el control externo, los estilos de interacción parental y las características del contexto en general, entre otras variables. Los niños en situación de vulnerabilidad social manifestaron diferencias en el patrón de respuestas R-I en relación con los niños sin riesgo; específicamente, se evidencia que cometen una mayor cantidad de errores y emplean un menor tiempo de latencia, indicadores ambos de mayor impulsividad cognitiva.

Una de las hipótesis al respecto es que el alto grado de impulsividad podría deberse a dificultades en el uso del lenguaje interno para guiar las conductas, consecuencia, a su vez, de las escasas interacciones sociales que condicionan el desarrollo de la autorregulación. Según Díaz, Winsler, Atencio y Harbes (1992), en algunos niños impulsivos las dificultades no se deberían a una falta de lenguaje interno, sino a una menor maduración y a un uso inadecuado del mismo, para autorregular las conductas. En este sentido, las estrategias de intervención para reducir la impulsividad se destinan a internalizar el lenguaje privado ya existente o a utilizarlo de un modo más efectivo, de manera que permita guiar las conductas (Díaz \& Berk, 1995). Asimismo, la impulsividad podría explicarse por la menor estimulación cognitiva en los primeros años de vida, característica común a los contextos de pobreza. Esto sería consistente con lo planteado por Olson et al. (1990) quienes señalaron que las interacciones madre-hijo enriquecidas cognitivamente, se asocian con capacidades futuras de control de impulsividad y la habilidad para postergar la gratificación.

TABLA 3

Comparación de puntajes obtenidos respecto a la Impulsividad y la Planificación entre niños con y sin vulnerabilidad social después de la intervención en el grupo experimental

\begin{tabular}{|c|c|c|c|c|c|c|c|c|c|c|c|}
\hline \multirow[t]{2}{*}{ Funciones } & \multicolumn{2}{|c|}{$\begin{array}{c}\text { Riesgo } \\
\text { experimental }\end{array}$} & \multicolumn{2}{|c|}{$\begin{array}{l}\text { Control con } \\
\text { riesgo }\end{array}$} & \multicolumn{2}{|c|}{ Control sin riesgo } & \multirow{2}{*}{$\begin{array}{c}F \\
(2,107)\end{array}$} & \multirow[t]{2}{*}{$P$} & \multirow{2}{*}{ M1-M2 } & \multirow{2}{*}{ M1-M3 } & \multirow{2}{*}{ M2-M3 } \\
\hline & M1 & DE1 & M2 & DE2 & M3 & DE3 & & & & & \\
\hline Impulsi & -0.8968 & 1.893 & 1.71 & 1.46 & -0.0056 & 1.53 & 18.049 & 0.000 & 0.000 & 0.045 & 0.001 \\
\hline - Errores & 28.96 & 12.82 & 39.91 & 10.65 & 31.71 & 9.188 & 7.275 & 0.001 & 0.001 & 0.754 & 0.019 \\
\hline - Latencia & 286.861 & 120.9 & 100.153 & 72.37 & 192.60 & 121.33 & 21.587 & 0.000 & 0.000 & 0.001 & 0.008 \\
\hline anificación & 20.87 & 4.19 & 16.18 & 4.99 & 22.34 & 2.56 & 18.609 & 0.000 & 0.000 & 0.234 & 0.0 \\
\hline
\end{tabular}

Fuente: elaboración propia 
Los resultados respecto a los efectos de la intervención sobre el estilo cognitivo, apuntan a la eficacia de los programas de intervención para aumentar la disposición reflexiva integrados a la tarea curricular áulica. En el grupo que recibió la intervención, se evidenció una disminución en la cantidad de errores y un aumento en los tiempos de latencia a un nivel similar al del grupo control sin riesgo. Es necesaria la existencia de este patrón de respuesta, ya que la sola presencia de un aumento de la latencia no constituye por sí misma un indicador de mayor reflexividad. Al mismo tiempo, se debe dar una disminución en la cantidad de errores, ya que el objetivo de la intervención consiste no sólo en lograr que los niños empleen más tiempo en dar una respuesta, sino que además lo utilicen eficazmente.

El carácter plástico del estilo cognitivo nos indica que se debe prestar especial atención desde edades muy tempranas y que se deben volver a pensar las praxis educativas, incorporando estrategias de intervención fácilmente aplicables al ámbito escolar, para aumentar la reflexividad. De esta manera, se plantea la importancia de evaluar el constructo R-I en la escuela, más aún en poblaciones en condiciones de vulnerabilidad social, por el alto grado de impulsividad encontrado y la evidencia, tanto teórica como empírica, a favor de la posibilidad de aumentar la reflexividad mediante un entrenamiento sistematizado (Richaud de Minzi, 2008a).

La planificación es una habilidad cognitiva que requiere de la capacidad de anticipar y preveer consecuencias, y de controlar y monitorear el curso de las acciones para alcanzar un objetivo. En este sentido, se considera que la planificación se encuentra relacionada con el estilo cognitivo, ya que si la construcción de planes requiere de control y autorregulación, se debería beneficiar del uso de un pensamiento reflexivo y de un estilo analítico frente al impulsivo. La capacidad de los niños para planificar reveló diferencias significativas según la vulnerabilidad social. Los niños provenientes de sectores marginales completaron una menor cantidad de laberintos y cometieron más errores, evidenciados por una mayor tendencia a entrar en pasajes sin salida. Una posible explicación al respecto es que la escasa estimulación que provee el entorno y la falta de regulación e interacciones verbales por parte del adulto, condicionarían el desarrollo de la autorregulación y la capacidad de planificación. Diversos estudios han indicado cómo los niveles de autocontrol no estarían influidos únicamente por factores biológicos (Barkley, 1997; Beaver, Wright, Delicia \& Vaughn, 2008), sino que estarían asimismo determinados por influencias sociales, como los factores familiares, las condiciones del barrio y el contexto escolar (Gottfredson \& Hirschi, 1990; Hay, 2001; Turner, Piquero \& Pratt, 2005). Asimismo, se ha señalado la importancia de la interacción verbal madrehijo en el desarrollo de la planificación (Gauvain, 2001; Gauvain \& Pérez, 2008) y en el desarrollo del sentido de temporalidad (Hudson, 2002). Esto sugiere que las características estimulares y la interacción adulto-niño influiría en el desarrollo de esta habilidad. Debido a que estudios previos han informado algunas diferencias respecto al estilo de la interacción cognitiva según el nivel socioeconómico (Ninio, 1980; Peralta de Mendoza, 1997; Peralta de Mendoza \& Salsa, 2001) y la escolaridad materna (De Tejada \& Otálora, 2006) el estilo de interacción cognitiva podría mediar la asociación entre la pobreza y el desarrollo cognitivo.

Respecto a los efectos de la intervención sobre la capacidad de planificación, los resultados indican un aumento de esta función a favor del grupo que recibió el entrenamiento. El grupo experimental aumentó la capacidad de planificación en relación con el grupo control con riesgo y obtuvo un nivel de desempeño similar al del grupo control sin riesgo. $\mathrm{Al}$ analizar conjuntamente los efectos de la intervención sobre la R-I y la capacidad de planificación, se puede presumir razonablemente la existencia de una relación entre ambas variables. Esta relación se explica en parte por los procesos cognitivos que se ponen en juego en la realización de ambas tareas, ya que, por un lado, para resolver problemas definidos por la incertidumbre se requiere no solo del empleo de estrategias analíticas sino de la elaboración de planes eficaces y, por otro lado, la capacidad de hacer planes se debería beneficiar 
de un estilo analítico y reflexivo. Además, tanto el uso de estrategias analíticas como la capacidad de hacer planes requiere de control atencional y de un lenguaje autodirigido y autorregulado. Esto permite inferir que las estrategias cognitivas empleadas en el presente estudio, tendientes a favorecer la autorregulación de la conducta y el uso de un pensamiento reflexivo, son igualmente efectivas para estimular tanto la capacidad de planificación como la disposición reflexiva.

Teniendo en cuenta lo desarrollado, el hecho de que el desempeño en ciertas funciones ejecutivas como la planificación y la reflexividad cognitiva mejore notablemente mediante el entrenamiento sistematizado, nos lleva a volver a pensar en el papel crítico de la experiencia en el desarrollo de estas funciones. Dowsett y Livesey (2000) sugieren que, si bien son numerosos los estudios sobre los efectos del desarrollo neural (maduración) en el perfeccionamiento del desempeño ejecutivo, hay una falta de investigación respecto a los efectos de la experiencia en los procesos ejecutivos.

Es importante indicar que la asociación entre el desempeño cognitivo y la pobreza, estaría mediada por las variables que delimitan a la pobreza. Estudios previos han sugerido que diferentes variables asociadas a la pobreza tales como: (a) la presencia de mayores conductas de riesgo para la salud física y mental (Adler et al., 1994); (b) la deprivación económica (Duncan, Brooks-Gunn, \& Klebanov, 1994); (c) la falta estimulación cognitiva en el hogar (Guo \& Harris, 2000); (d) la menor calidad del entorno en el hogar que proveen las madres (Baharudin \& Luster, 1998); (e) la mayor exposición al estrés en el ambiente familiar y social (Dearing, 2008) y (f) las características ambientales en general (Evans, 2004), mediarían la asociación entre la pobreza y el desarrollo del niño

Por último, señalar que si bien los cambios posintervención apuntan a que sería la falta de experiencia y de entrenamiento especifico la que ocasionaría diferencias de funcionamiento cognitivo en las poblaciones de niños en riesgo, esta falta de estimulación prolongada en el tiempo podría desencadenar trastornos del desarrollo futuros. Diferentes autores han señalado cómo los niños expuestos a condiciones de pobreza, tienden a mostrar una declinación en su desarrollo cognitivo, motor y socioemocional (Brooks-Gunn \& Duncan, 1997; Brooks-Gunn, Duncan, Klevanov \& Sealand, 1993; Dearing, 2008; Grantham-McGregor et al., 2007), menores logros académicos en el futuro (Brooks-Gunn \& Duncan, 1997) y un menor funcionamiento cognitivo en la adultez (Kaplan et al., 2001). Asimismo, se ha sugerido que los niños que viven en situación de pobreza tienen más probabilidades de presentar problemas y conflictos en la esfera social y emocional. Por ejemplo, estudios previos han indicando que los niños y adolescentes que crecen en la pobreza de comunidades urbanas presentan un mayor riesgo de infringir leyes y normas sociales (Anderson, 1999; Wilson, 1987).

Es decir que el menor desempeño cognitivo evidenciado, aunque reversible, podría perpetuarse en el tiempo alterando de manera más o menos definitiva la neurodinámica cortical. Por lo tanto, y teniendo en cuenta que el desarrollo del córtex prefrontal continúa durante la adolescencia (Diamond, 2002), consideramos importante la intervención en las poblaciones en riesgo, valiéndonos de la ventana de oportunidades que ofrece el desarrollo cerebral y que convenientemente coincide con la etapa escolar (Richaud de Minzi, 2008b). Los estudios sugieren que la intervención intensiva en niños en riesgo tiene efectos duraderos en el desarrollo cognitivo y académico (Campbell, Pungello, Miller-Johnson, Burchinal \& Ramey, 2001) y que en la reducción del impacto de la pobreza sobre el desarrollo cognitivo de los niños, las intervenciones más importantes son las que ocurren en la temprana infancia (Brooks-Gunn \& Duncan, 1997).

\section{Referencias}

Aber, J. L., Bennet, N. G., Conley, D. C. \& Li, J. (1997). The effects of poverty on child health and development. Annual Review of Public Health, 18, 463-483.

Adler, N. E., Boyce, T., Chesney, M. A., Cohen, S., Folkman, S., Kahn, R. L. \& Syme, S. L. (1994). Socioeconomic status and health: The challenge of the gradient. American Psychologist, 49, 15-24. 
Anderson, E. (1999). Code of the street: Decency, violence, and the moral life of the inner city. New York: Norton.

Arán Filippetti, V. \& Richaud de Minzi, M. C. (2008). P.E.C.E. Programa de Estimulación Cognitivo Escolar: Cuaderno de ejercicios nivel I. Buenos Aires: Ediciones CIIPME-CONICET.

Baharudin, R. \& Luster, T. (1998). Factors related to the quality of the home environment and children's achievement. Journal of Family Issues, 19, 375-403.

Baker-Sennett, J., Matusov, E. \& Rogoff, B. (1992). Sociocultural processes of creativity and planning in children's playcrafting. In P. Light \& G. Butterworth (Eds.), Context and cognition: Ways of learning and knowing (pp. 93-114). New York: Harvester Wheatsheaf.

Baker-Sennett, J., Matusov, E. \& Rogoff, B. (1993). Planning as developmental process. In H. W. Reese (Ed.), Advances in child development and behavior (Vol. 24, pp. 253-281). New York: Academic Press.

Barkley, R. A. (1997). Behavioral inhibition, sustained attention, and executive functions: Constructing a unifying theory of ADHD. Psychological Bulletin, 121, 65-94.

Beaver, K. M., Wright, J. P., DeLisi, M. \& Vaughn, M. G. (2008). Genetic influences on the stability of low self-control: Results from a longitudinal sample of twins. Journal of Criminal Justice, 36, 478-485.

Brooks-Gunn, J. \& Duncan, G. J. (1997). The Effects of Poverty on Children. The Future of Children, 7, 55-71.

Brooks-Gunn, J., Duncan, G. J., Klebanov, P. K. \& Sealand, N. (1993). Do neighborhoods influence child and adolescent development? American Journal of Sociology, 99, 353-395.

Buela-Casal, G., Carretero-Dios, H. \& De los SantosRoig, M. (2001). Análisis del constructo reflexividad-impulsividad: del Matching Familiar Figures Test (MFFT) al MFFT 20. Análisis y Modificación de Conducta, 27, 29-70.

Bush, G., Luu, P. \& Posner, M. I. (2000). Cognitive and emotional influences in anterior cingulate cortex. Trends in Cognitive Sciences, 4, 215-222.

Carines, E. \& Cammock, T. (1978). Development of a more reliable version of the Matching Familiar Figures Test. Developmental Psychology, 14, 555-560.
Campbell, F. A., Pungello, E. P., Miller-Johnson, S., Burchinal, M. \& Ramey, C. T. (2001). The development of cognitive and academic abilities: Growth curves from an early childhood educational experiment. Developmental Psychology, 37, 231-242.

Damasio, A. R. (1999). The feeling of what happens: Body and emotion in the making of consciousness. New York: Harcourt Brace.

Davis, M. (1992). The role of the amygdala in conditioned fear. In J. P. Aggleton (Ed.), The amygdala: neurobiological aspects of emotion, memory, and mental dysfunction (pp. 255-306). New York: Wiley-Liss.

Dearing, E. (2008). Psychological costs of growing up poor. Annals of the New York Academy of Sciences, 1136, 324-332.

De Tejada, M. \& Otálora, C. (2006). Estimulación cognitiva de madres del sector popular. Investigación y Postgrado, 21, 43-68.

Diamond, A. (2002). Normal development of prefrontal cortex from birth to young adulthood: cognitive functions, anatomy, and biochemistry. In D.T. Stuss \& R.T. Knight (Eds.), Principles of frontal lobe function (pp. 466-503). London: Oxford University Press.

Díaz, R. M, Winsler, A., Atencio, D. J. \& Harbers, K. (1992). Mediation of self regulation trough the use of private speech. International Journal of Cognitive Education and Mediated Learning, 2, 1-13.

Díaz, R. M. \& Berk, L. E. (1995). A Vygotskian critique of self-instructional training. Development and Psychopathology, 7, 369-392.

Dowsett, S. M. \& Livesey, D. J. (2000). The development of inhibitory control in preschool children: Effects of "executive skills" training. Developmental Psychobiology, 36, 161-174.

Duncan, G., Brooks-Gunn, J. \& Klebanov, P. (1994). Economic deprivation and early childhood development. Child Development, 65, 296-318.

Egeland, B. (1974). Training impulsive children in the use of more efficient scanning techniques. Child Development, 45, 165-171.

Evans, G. W. (2004). The environment of childhood poverty. American Psychologist, 59, 77-92.

Farah, M., Shera, D., Savage, J., Betancourt, L., Giannetta, J., Brodsky, N. et al. (2006). Childhood 
poverty: Specific associations with neurocognitive development. Brain research, 1110, 166-174.

Fernández-Millán, J. M., Pérez Mañez, D. \& Carrasco Salmerón, S. (2002). Impulsividad cognitiva en menores maltratados: factores influyentes. Universitas Psychologica, 1 (2), 21-26.

Flinn, M. V. \& England, B. G. (1997). Social economics of childhood glucocorticoid stress response and health. American Journal of Physical Anthropology, 102, 33-53.

Gauvain, M. (2001). The social context of cognitive development. New York: Guilford Press.

Gauvain, M. \& Pérez, S. M. (2008). Mother-child planning and child compliance. Child Development, 79, 761-775.

Gargallo, B. (1993a). ¿Es posible modificar la impulsividad en el aula? Programas de acción educativa. Revista de Educación, 301, 245-268.

Gargallo, B. (1993b). Basic variables in ReflectionImpulsivity: A training program to increase reflectivity. European Journal of Psychology of Education, 8 (2), 151-167.

Gottfredson, M. \& Hirschi, T. (1990). A general theory of crime. Palo Alto, CA: Stanford University Press.

Grantham-McGregor, S., Cheung, Y., Cueto, S., Glewwe, P., Richter, L. \& Strupp, B. (2007). Developmental potential in the first 5 years for children in developing countries. Lancet, 369, 60-70.

Guo, G. \& Harris, K. M. (2000). The mechanisms mediating the effects of poverty on children's intellectual development. Demography, 37 (4), 431-447.

Hay, C. (2001). Parenting, self-control, and delinquency: A test of self-control theory. Criminology, 39, 707-736.

Hudson, J. A. (2002). "Do we know what we're going to do this summer?" Mothers' talk to young children about future events. Journal of Cognition and Development, 3, 49-71.

Kagan, J. (1965). Matching Familiar Figures Test. Cambridge: Hardvard University.

Kagan, J., Rosman, B., Day, D., Albert, J., \& Phillips, W. (1964). Information processing in the child: Significance of analytic and reflective attitudes. Psychological Monographs, 78 (1, Whole No. 587)
Kaplan, G., Turrel, G., Lynch, J., Everson, S., Helkala, E. L. \& Salonen, J. T. (2001). Chilhood socioeconomic position and cognitive function in adulthood. International Journal of Epidemiology, 30, 256-263.

Larson, M., Gunnar, M. \& Hertzgaard, L. (1991). The effects of morning naps, car trips, and maternal separation on adrenocortical activity in human infants. Child Development, 62, 362-372.

LeDoux, J. (1996). The emotional brain. New York: Simon \& Schuster.

Lewis, M. \& Thomas, D. (1990). Cortisol release in infants in response to inoculation. Child Development, 61, 50-59.

Lezak, M. (1995). Neuropsychological assessment (3 ${ }^{\text {rd }}$ ed.). New York: Oxford University Press.

Lipina, S. J., Martelli, M. I., Vuelta, B. L., Injoque-Ricle, I. \& Colombo, J. A. (2004). Pobreza y desempeño ejecutivo en alumnos preescolares de la ciudad de Buenos Aires (Republica Argentina). Interdisciplinaria, 21 (2), 153-193.

Lupien, S. J., King, S., Meaney, M. J. \& McEwen, B. S. (2001). Can poverty get under your skin? Basal cortisol levels and cognitive function in children from low and high socioeconomic status. Developmental and Psychopathology, 13, 653-676.

Luria, A. R. (1961). The role of speech in the regulation of normal and abnormal behavior. New York: Liveright.

McLanahan, S. S., Astone, N. M. \& Marks, N. (1991). The role of mother-only families in reproducing poverty. In A. C. Huston (Ed.), Children in poverty: Child development and public policy (pp. 51-78). New York: Cambridge University Press.

Meichenbaum, D. H. \& Goodman, J. (1971). Training impulsive children to talk to themselves: A means of developing self-control. Journal of Abnormal Psychology, 77, 115-126.

Miranda, A., Presentación, M. J. \& Jarque, S. (1999). La intervención con estudiantes con TDAH: hacia un enfoque contextualizado y multidisciplinar. En J. N. García Sánchez (Coord.), Intervención psicopedagógica en los trastornos del desarrollo (pp. 303-318). Madrid: Psicología Pirámide.

Miranda, A., Roselló, M. B. \& Soriano, M. (1998). Estudiantes con deficiencias atencionales. Valencia: Promolibro. 
Ninio, A. (1980). Picture book reading in mother-infant dyads belonging to two subgroups in Israel. Child Development, 51, 587-590.

Noble, K. G., Norman, M. F. \& Farah, M. J. (2005). Neurocognitive correlates of socioeconomic status in kindergarten children. Developmental Science, 8, 74-87.

Olson, S. L., Bates, J. E. \& Bayles, K. (1990). Early antecedents of childhood impulsivity: The role of parent-child interaction, cognitive competence, and temperament. Journal of Abnormal Child Psychology, 18, 317-334.

Peralta de Mendoza, O. A. (1997). Estilos de interacción cognitiva materno-infantil en una situación de resolución de problemas en función del nivel socioeconómico y de la edad del niño. Infancia y Aprendizaje, 20, 85-98.

Peralta de Mendoza, O. A. \& Salsa A. M. (2001). Interacción materno-infantil con libros con imágenes en dos niveles socioeconómicos. Infancia y Aprendizaje, 24, 325-339.

Posner, M. I. \& Rothbart, M. K. (2000). Developing mechanisms of self-regulation. Development and Psychopathology, 12, 427-441.

Radziszewska, B. \& Rogoff, B. (1988). Influence of adult and peer collaborators on children's planning skills. Developmental Psychology, 24, 840-848.

Ramey, C. T. \& Campbell, F. A. (1991). Poverty, early childhood education, and academic competence: The Abecedarian experience. In A. C. Huston (Ed.), Children in poverty: Child development and public policy (pp.190-221). New York: Cambridge University Press.

Richaud de Minzi, M. C. (2008a, noviembre). Niños en riesgo por pobreza. Aportes para una educación que respete su diversidad. Conferencia internacional invitada de apertura, Primer Congreso Internacional de Psicología y Pobreza, Huancayo, Perú.

Richaud de Minzi, M. C. (2008b, julio). How to reinforce psychological resources in children at risk by poverty. In M. C. Richaud de Minzi (Org.), Invited Symposium Development of Psychological and Biological Resources in Children at Risk, XXIX International Congress of Psychology, Berlin, Germany.

Servera- Barceló, M. \& Galván Pascual, M. (2001). Problemas de impulsividad e inatención en el niño. Propuestas para su evaluación. Madrid: Ministerio de educación. Centro de Investigación y Documentación educativa (C.I.D.E.).

Shonkoff, J. P. \& Phillips, D. A. (Eds.). (2000). From neurons to neighborhoods: The science of early child development. Washington, DC: National Academy Press.

Turner, M. G., Piquero, A. R. \& Pratt, T. C. (2005). The school context as a source of self-control. Journal of Criminal Justice, 33, 327-339.

Unterrainer, J. M., Rahm, B., Kaller, C. P., Ruff, C. C., Spreer, J., Krause, B. J. et al. (2004). When planning fails: Individual differences and error-related brain activity in problem solving. Cerebral Cortex December, 14, 1390-1397.

Wilson, W. J. (1987). The truly disadvantaged: The inner city, the underclass, and public policy. Chicago: University of Chicago Press.

Winsler, A., Díaz, R. M., McCarthy, E. M., Atencio, D. J. \& Chabay, L. A. (1999). Mother-child interaction, private speech, and task performance in preschool children with behavior problems. Journal of Child Psychology and Psychiatry, 40, 891-904. 